\title{
Practical guidelines in the application of response evaluation criteria for solid tumours (RECIST) in oncology imaging
}

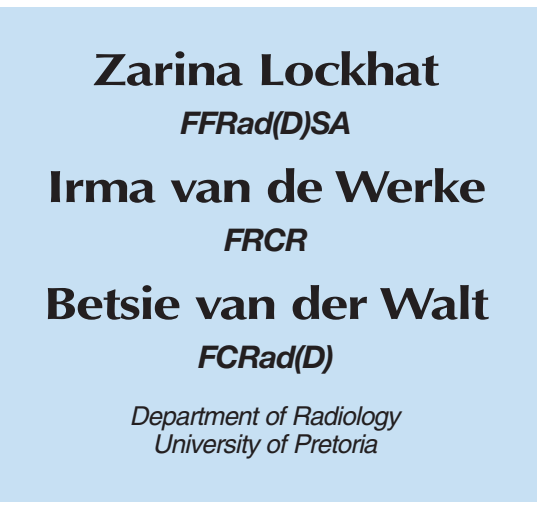

The World Health Organization (WHO), the National Cancer Institute and the European Organisation for Research and Treatment of Cancer have recently adopted a new set of tumour response criteria Response Evaluation Criteria for Solid Tumours (RECIST). Monitoring response of tumours to treatment is an integral and increasingly important function of radiologists working in oncologic imaging. Imaging studies play a pivotal, objective role in quantifying tumour response to a variety of physical and pharmaceutical treatments. The RECIST criteria have been introduced to unify response assessment criteria, to define how to choose measurable lesions, and to enable the use of new imaging technologies (spiral computed tomography (CT) and magnetic resonance imaging (MRI)).

\section{Definitions and measurements}

The four categories of response are: (i) complete response (CR); (ii) partial response (PR); (iii) stable disease (SD); and (iv) progressive disease (PD).

RECIST criteria rely on size change of lesions to make response assessments. Unidimensional measurements and the sum of the longest diameters are now used, instead of the conventional method using two measurements and the sum of the products.

At baseline, lesions are to be categorised as measurable or non-measurable. Measurable lesions are defined as those that can be measured accurately in at least one diameter, that is $\geq 20 \mathrm{~mm}$ using conventional imaging techniques (including incremental CT) or $\geq 10 \mathrm{~mm}$ using spiral CT.

Non-measurable lesions are discrete lesions with smaller dimensions. These lesions include bony metastases, leptomeningeal disease, ascites, pleural/pericardial effusions, inflammatory breast cancer, lymphangitis carcinomatosa (cutis/pulmonis), abdominal masses that are not confirmed and followed by imaging techniques, heavily calcified and cystic/ necrotic lesions.

Interestingly, tumour lesions situated in a previously irradiated area may also not be considered as measurable disease. The term 'evaluable' which refers to lesions that can be viewed but cannot be measured, has been dropped.

After establishing that measurable disease exists, it is necessary to document 'target' lesions and non-target lesions. Measurable lesions up to a maximum of five lesions per organ and 10 lesions in total, representative of all involved organs, should be identified as 'target lesions'. These target lesions should be selected on the basis of size and suitability for accurate repeated measurements. A sum of the longest diameter of all target lesions constitutes the baseline sum longest diameter.

Changes in the sum of the longest diameter are to be used to categorise 'target tumour response'. Non-target lesions need not be measured on follow-up studies but any change should be noted. Final response should take into account changes in both target and non-target lesions as well as noting the presence or absence of new disease. It is noteworthy that for stable disease and for progressive disease, the pre-treatment study no longer serves as the baseline study, instead the reference study is one where the smallest sum of the longest diameter was recorded.

Baseline evaluations are to be performed as close as possible to the beginning of treatment, but not more 
Table I. Definition of best response according to WHO or RECIST criteria

\begin{tabular}{lll}
\hline Best response & WHO change in sum of products & $\begin{array}{l}\text { RECIST change in the sum of the } \\
\text { longest diameter }\end{array}$ \\
\hline Complete response (CR) & $\begin{array}{l}\text { Disappearance of all target lesions } \\
\text { without any residual lesion; confirmed } \\
\text { at } 4 \text { weeks } \\
\begin{array}{l}50 \% \text { or more decrease in target lesions, } \\
\text { without a 25\% increase in any one } \\
\text { target lesion; confirmed at } 4 \text { weeks }\end{array}\end{array}$ & $\begin{array}{l}\text { Disappearance of all } \\
\text { target lesions; confirmed at } 4 \text { weeks }\end{array}$ \\
Partial response (PR) & $\begin{array}{l}\text { At least 30\% reduction in the sum } \\
\text { of the longest diameter of target } \\
\text { lesions, taking as reference the } \\
\text { baseline study; confirmed at } \\
\text { Stable disease (SD) }\end{array}$ & $\begin{array}{l}\text { Neeks } \\
\text { Neither PR nor PD criteria are met, } \\
\text { taking as reference the smallest sum of } \\
\text { the longest diameter recorded since } \\
\text { treatment started }\end{array}$ \\
& $\begin{array}{l}\text { At least 20\% increase in the sum } \\
\text { of the longest diameter of target } \\
\text { lesions, taking as reference the } \\
\text { Progressive disease (PD) }\end{array}$ & $\begin{array}{l}\text { smallest sum of the longest diameter } \\
\text { recorded since treatment started or } \\
\text { appearance of new lesions }\end{array}$
\end{tabular}

than 4 weeks before treatment starts. With re-evaluation studies there is flexibility — it is recommended that follow-up every other cycle be performed - every 6 - 8 weeks.

An end of treatment study enables overall treatment response assessment. In patients with partial response (PR) or complete response (CR) confirmatory imaging is required at 4 weeks after the criteria for CR or PR have been met.

\section{Practical imaging recom- mendations}

The same method of assessment and same technique should be used to characterise each identified and reported lesion at baseline and during follow-up.

Imaging-based evaluation is pre- ferred to evaluation by clinical examination when both methods have been used to assess the anti-tumour effect of a treatment.

\section{Chest X-ray}

Except for the chest radiograph, the role of radiography is not that important. Lesions are considered measurable when they are clearly defined and surrounded by aerated lung. However CT is preferable. Radiographs cannot be used to assess bone lesions because bony metastases are classified as non-measurable lesions.

\section{Ultrasound}

This is not used routinely to assess response of lesions because it is operator-dependent and cannot be reproduced for independent review at a later date. However ultrasound may be used as an alternative to clinical measurement — for superficially palpable lymph nodes, subcutaneous lesions and thyroid nodules.

\section{CT and MRI}

These are the best currently available modalities and the most reproducible.

For MRI assessment the same anatomical plane is used for subsequent examinations. There are no specific sequence recommendations.

For CT, when choosing measurable lesions, the basic rule to be followed is that the minimum size should be not less than double the slice thickness. This is to minimise partial volume averaging that can lead to underestimation of lesion size. The longest diameter of the target lesion should be 
obtained in the axial plane only.

For spiral CT the minimum size of a lesion may be $10 \mathrm{~mm}$ provided that a $10 \mathrm{~mm}$ collimation is used and reconstructions are at $5 \mathrm{~mm}$ intervals. For conventional incremental CT, the minimum size of lesions should be 20 $\mathrm{mm}$ with the use of contiguous 10 mm thick slices.

Contrast medium usage is recommended. Oral contrast medium is used routinely - some studies have shown that water is a better contrast agent when evaluating stomach and bowel lesions.

IV contrast is also used routinely some lesions become measurable only after IV contrast administration; however contrast may not be necessary when evaluating discrete lung disease.

Another important recommenda- tion is that all images should be filmed, not just selected images of target lesions. Lesions should be measured on the same window setting at each examination.

\section{Conclusion}

With the introduction of RECIST criteria the role of imaging has become more important. CT examinations are performed at an increasing frequency, however the use of plain radiographs and ultrasound has declined.

The method of assessing lesions, i.e. unidimensional measurement instead of bidimensional diameters has now been recommended. The measurement of lesions has previously been regarded as laborious and time -consuming. Thus radiologists should be familiar with the RECIST criteria so that measurements are easier to make, more accurate, relevant and less time-consuming to guide clinical decision-making.

\section{Acknowledgement}

We would like to thank Annelize Gates for her assistance in the preparation of this article.

\section{References}

1. Padhani AR, Ollivier L. The RECIST criteria: Implications for diagnostic radiologists. $\mathrm{Br} \mathrm{J}$ Radiol 2001: 74: 983 -986.

2. McHugh K, Kao S. Response evaluation criteria in solid tumours (RECIST) problems and need for modifications in paediatric oncology? $\mathrm{Br} \mathrm{J}$ Radiol 2003: 76: 433-436.

3. Padhani AR, Husbano JE. Are current tumour response criteria relevant for the 21 st century? Br J Radiol 2000: 73: 1031-1033.

4. Therasse P, Arbuck SG, et al. Special article: New guidelines to evaluate the response to treatment in solid tumours. J Natl Cancer Institute 2002; 92: 205-215. 